\title{
Nonequilibrium Green's function method for thermal transport in junctions
}

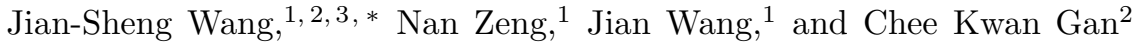 \\ ${ }^{1}$ Center for Computational Science and Engineering, \\ and Department of Physics, National University of Singapore, Singapore 117542 \\ ${ }^{2}$ Institute of High Performance Computing, 1 Science Park Road, Singapore 117528 \\ ${ }^{3}$ Singapore-MIT Alliance, 4 Engineering Drive 3, Singapore 117576
}

(Dated: 9 January 2007)

\begin{abstract}
We present a detailed treatment of the nonequilibrium Green's function method for thermal transport due to atomic vibrations in nanostructures. Some of the key equations, such as self-energy and conductance with nonlinear effect, are derived. A self-consistent mean-field theory is proposed. Computational procedures are discussed. The method is applied to a number of systems including one-dimensional chains, a benzene ring junction, and carbon nanotubes. Mean-field calculations of the Fermi-Pasta-Ulam model are compared with classical molecular dynamics simulations. We find that nonlinearity suppresses thermal transport even at moderately high temperatures.

PACS numbers: 05.60.Gg, 44.10.+i, 63.22.+m, 65.80.+n
\end{abstract}

\section{INTRODUCTION}

Fourier's law describes the transport of heat in a macroscopic object. The calculation of the thermal conductivity that appears in the Fourier's law is fundamental and important in understanding the properties of materials. Such a calculation must be grounded upon quantum mechanics with phonons as basic quasi-particles. This is a non-trivial task and was successfully carried out many years ago by Peierls [1, 2] using the Boltzmann equation for phonons. Molecular dynamic (MD) simulation is another approach. However, MD results are not correct at low temperatures as it is purely a classical approach.

The early works are mostly concerned with bulk materials with many degrees of freedom and periodic lattices [3]. In recent years, more attentions are paid to heat transport in small or low-dimensional systems such as carbon nanotubes and molecular junctions [4, 5]. In nano- to meso-scales, new features come in. In most of these conditions, the discreteness of the atom is important. The concept of phonons developed for the periodic lattices is somewhat difficult to apply if a system does not possess translational invariance, such as a nano-junction. In such situation, the Boltzmann-Peierls' approach is not applicable.

There are several alternative methods that have overcome the above problems. First, the Landauer formula [6] is a simple and clear description of the heat transport in purely ballistic regime at low temperatures. There are also a few other approaches [7, 8] with different approximations such as the description based on density matrices. One of the features of the existing theories is that they work in either the ballistic regime or diffusive regime, but not both. It is rather difficult to have a complete theory that can encompass both regimes, apart from phenomenological treatments $[9,10]$.

*http://staff.science.nus.edu.sg/ phywjs/
The nonequilibrium Green's function formalism described in this article is a serious attempt to be such a complete theory. This theory is certainly "first principles" given the atomic potentials. Several similar formulations have already been done at the elastic level without nonlinear interactions [11, 12, 13, 14]. In Ref. 15], we presented a new formulation with the nonlinear interaction treated systematically. Mingo also gave a similar result [16] for the nonlinear interactions. Our approach is essentially a generalization of the nonequilibrium Green's function method in electronic transport [17, 18], with fermions replaced by bosons as basic entities. Although the techniques have been extensively described in the literature, most of them are centered around fermions. In this paper, we give a description of the method, emphasizing on both the theoretical formulation and computational implementation. We begin with an elementary introduction of the Green's functions, including the contour-ordered Green's functions. We then discuss equations of motion of the Green's functions, Feynman diagrammatic expansion, and Dyson equations. We discuss the heat current and derive a formula for effective transmission when there are nonlinear interactions. After the introduction of the method, we present results of one-dimensional (1D) chains, benzene ring, and carbon nanotubes, and discuss some of the interesting features in such systems.

\section{NONEQUILIBRIUM GREEN'S FUNCTION METHOD}

\section{A. Definition of Green's functions}

The nonequilibrium Green's function methods are discussed in Refs. [18, 19], and equilibrium Green's functions are explained in many textbooks such as Refs. [20, 21]. For completeness, we introduce our notations and give a quick review of the definitions and properties of various Green's functions in this subsection. We define the 
retarded Green's function as

$$
G^{r}\left(t, t^{\prime}\right)=-\frac{i}{\hbar} \theta\left(t-t^{\prime}\right)\left\langle\left[u(t), u\left(t^{\prime}\right)^{T}\right]\right\rangle,
$$

where the column vector $u$ with component $u_{j}$ is not the usual displacement operator from equilibrium but a renormalized one, i.e., $u_{j}=x_{j} \sqrt{m_{j}}$, such that the kinetic energy is of the form $(1 / 2) \dot{u}^{T} \dot{u}$. The superscript $T$ stands for matrix transpose. The square brackets are the commutators. $G^{r}$ is a square matrix with elements $G_{j k}^{r}\left(t, t^{\prime}\right)=-(i / \hbar) \theta\left(t-t^{\prime}\right)\left\langle\left[u_{j}(t), u_{k}\left(t^{\prime}\right)\right]\right\rangle$. The physical dimension of $G^{r}$ is time. By definition, $G^{r}\left(t, t^{\prime}\right)$ equals zero when $t \leq t^{\prime}$. In equilibrium or nonequilibrium steady states, the Green's function depends only on the difference in time, $t-t^{\prime}$. The Fourier transform of $G^{r}\left(t-t^{\prime}\right)=G^{r}\left(t, t^{\prime}\right)$ is defined as

$$
G^{r}[\omega]=\int_{-\infty}^{+\infty} G^{r}(t) e^{i \omega t} d t .
$$

Note that we use square brackets to delimit the argument for the Green's functions in frequency domain. The dimension of the retarded Green's function in frequency domain is time squared. The inverse transform is given by

$$
G^{r}(t)=\frac{1}{2 \pi} \int_{-\infty}^{+\infty} G^{r}[\omega] e^{-i \omega t} d \omega .
$$

For notational simplicity, we'll set $\hbar=1$. We can always get the final required expressions by a simple dimension analysis.

The rest of the definitions are the advanced Green's function

$$
G^{a}\left(t, t^{\prime}\right)=i \theta\left(t^{\prime}-t\right)\left\langle\left[u(t), u\left(t^{\prime}\right)^{T}\right]\right\rangle,
$$

the "greater than" Green's function

$$
G^{>}\left(t, t^{\prime}\right)=-i\left\langle u(t) u\left(t^{\prime}\right)^{T}\right\rangle
$$

the "less than" Green's function

$$
G^{<}\left(t, t^{\prime}\right)=-i\left\langle u\left(t^{\prime}\right) u(t)^{T}\right\rangle^{T},
$$

the time-ordered Green's function

$$
G^{t}\left(t, t^{\prime}\right)=\theta\left(t-t^{\prime}\right) G^{>}\left(t, t^{\prime}\right)+\theta\left(t^{\prime}-t\right) G^{<}\left(t, t^{\prime}\right)
$$

and anti-time-ordered Green's function

$$
G^{\bar{t}}\left(t, t^{\prime}\right)=\theta\left(t^{\prime}-t\right) G^{>}\left(t, t^{\prime}\right)+\theta\left(t-t^{\prime}\right) G^{<}\left(t, t^{\prime}\right) .
$$

The following linear relations hold both in frequency and time domains from the basic definitions:

$$
\begin{aligned}
G^{r}-G^{a} & =G^{>}-G^{<}, \\
G^{t}+G^{\bar{t}} & =G^{>}+G^{<}, \\
G^{t}-G^{\bar{t}} & =G^{r}+G^{a} .
\end{aligned}
$$

Also, the relations $G^{r}=G^{t}-G^{<}$and $G^{a}=G^{<}-G^{\bar{t}}$ are useful. Out of the six Green's functions, only three of them are linearly independent. However, in systems with time translational invariance, the functions $G^{r}$ and $G^{a}$ are Hermitian conjugate of one other:

$$
G^{a}[\omega]=\left(G^{r}[\omega]\right)^{\dagger}
$$

So in general nonequilibrium steady-state situations, only two of them are independent. In this paper, we consider them to be $G^{r}$ and $G^{<}$. There are other relations in the frequency domain as well:

$$
\begin{aligned}
G^{<}[\omega]^{\dagger} & =-G^{<}[\omega] \\
G^{r}[-\omega] & =G^{r}[\omega]^{*} \\
G^{<}[-\omega] & =G^{>}[\omega]^{T}=-G^{<}[\omega]^{*}+G^{r}[\omega]^{T}-G^{r}[\omega]^{*}
\end{aligned}
$$

The last two equations show that we only need to compute the positive frequency part of the functions.

In thermal equilibrium, there is an additional equation relating $G^{r}$ and $G^{<}$:

$$
G^{<}[\omega]=f(\omega)\left(G^{r}[\omega]-G^{a}[\omega]\right)
$$

where

$$
f(\omega)=\frac{1}{\exp (\beta \hbar \omega)-1}
$$

is the Bose-Einstein distribution function at temperature $T=1 /\left(k_{B} \beta\right)$. Equation (16) is obtained by writing the Green's functions as a sum of energy eigenstates (known as the Lehmann representation). Thus in equilibrium, there is only one independent Green's function; we take it to be $G^{r}$.

The contour-ordered Green's function is a convenient book-keeping to treat the different Green's functions in a concise notation. We can consider a contour-ordered Green's function as a function $G\left(\tau, \tau^{\prime}\right)$ with arguments $\tau$ and $\tau^{\prime}$ defined on the complex plane. The contour runs from $-\infty$ slightly above the real axis to $+\infty$ and loops back from $+\infty$ slightly below the real axis to $-\infty$. The contour-ordered Green's function can be mapped onto four different normal Green's functions by $G^{\sigma \sigma^{\prime}}\left(t, t^{\prime}\right)=$ $\lim _{\epsilon \rightarrow 0^{+}} G\left(t+i \epsilon \sigma, t^{\prime}+i \epsilon \sigma^{\prime}\right)$, where $\sigma= \pm(1)$, and $G^{++}=$ $G^{t}$ is the time-ordered Green's function, $G^{--}=G^{\bar{t}}$ is the anti-time-ordered Green's function, $G^{+-}=G^{<}$, and $G^{-+}=G^{>}$.

In a noninteracting harmonic system with a Hamiltonian

$$
H_{0}=\frac{1}{2} \dot{u}^{T} \dot{u}+\frac{1}{2} u^{T} K u,
$$

the retarded Green's function in the frequency domain is given by

$$
G^{r}[\omega]=\left((\omega+i \eta)^{2} I-K\right)^{-1},
$$

where $I$ is an identity matrix, and $\eta \rightarrow 0^{+}$. Adding a small $\eta$ helps to choose correctly the inverse Fourier 
transform integration path in the complex $\omega$-plane, so that the retarded Green's function has the required causal property, $G^{r}(t)=0$ for $t<0$. Equation (19) can be derived by an equation of motion method. We note that the retarded Green's function is a symmetric matrix since $K$ is symmetric, but this feature is not preserved with nonlinear interactions.

\section{B. Model and adiabatic switch-on}

Our system is a junction with a central region and two leads which serve as heat reservoirs. We treat the leads explicitly as quasi-one-dimensional periodic lattices. This setup is experimentally relevant and conceptually useful for computation. We consider nonconducting solid and treat only the vibrational degrees of freedom for heat transport. Let the (mass-normalized) displacement from some equilibrium position for the $j$ th degree of freedom in the region $\alpha$ be $u_{j}^{\alpha} ; \alpha=L, C, R$, for the left, center, and right regions, respectively. The quantum Hamiltonian is given by

$$
\mathcal{H}=\sum_{\alpha=L, C, R} H_{\alpha}+\left(u^{L}\right)^{T} V^{L C} u^{C}+\left(u^{C}\right)^{T} V^{C R} u^{R}+H_{n},
$$

where $H_{\alpha}=\frac{1}{2}\left(\dot{u}^{\alpha}\right)^{T} \dot{u}^{\alpha}+\frac{1}{2}\left(u^{\alpha}\right)^{T} K^{\alpha} u^{\alpha}, u^{\alpha}$ is a column vector consisting of all the displacement variables in region $\alpha$, and $\dot{u}^{\alpha}$ is the corresponding conjugate momentum. $K^{\alpha}$ is the spring constant matrix and $V^{L C}=$ $\left(V^{C L}\right)^{T}$ is the coupling matrix of the left lead to the central region; similarly for $V^{C R}$. We note that the dynamic matrix of the full linear system is

$$
K=\left(\begin{array}{lll}
K^{L} & V^{L C} & 0 \\
V^{C L} & K^{C} & V^{C R} \\
0 & V^{R C} & K^{R}
\end{array}\right) .
$$

The nonlinear part of the interaction will take the form

$$
H_{n}=\frac{1}{3} \sum_{i j k} T_{i j k} u_{i}^{C} u_{j}^{C} u_{k}^{C}+\frac{1}{4} \sum_{i j k l} T_{i j k l} u_{i}^{C} u_{j}^{C} u_{k}^{C} u_{l}^{C} .
$$

The quartic interaction is important in stablizing the system, as a purely cubic nonlinear interaction makes the energy unbounded from below.

We need to answer an important question: what is the distribution of the system? The distribution enters the definition of the Green's functions as a density matrix $\rho(0)$ for the average $\langle\cdots\rangle$. For an equilibrium problem, this is just the Boltzmann factor, but in a nonequilibrium situation, it is not known and must be computed in some way. The adiabatic switch-on gives us a clear conceptual framework how this problem can be solved, at least formally. We imagine that at $t=-\infty$ the system has three decoupled regions, each at separate temperatures, $T_{L}, T_{C}$, and $T_{R}$. The nonlinear interactions are turned off. The Green's functions $g^{\alpha}$ are known and take the form of Eq. (19). The couplings $V^{L C}$ and $V^{C R}$ are then

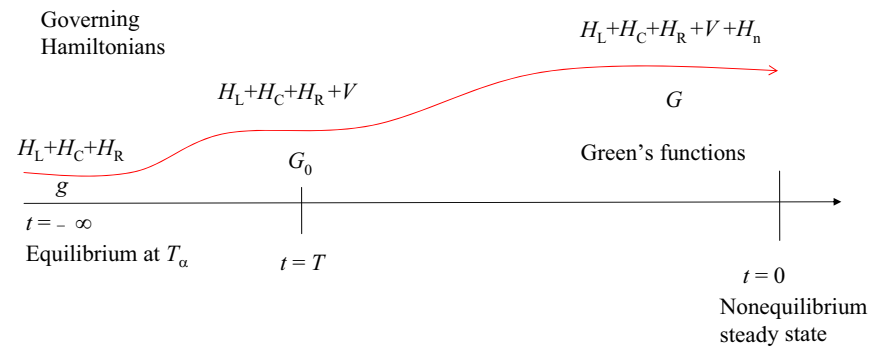

FIG. 1: A schematic to illustrate the two adiabatic switchons.

turned on slowly, and a steady state of the linear system is established at some time $T \ll 0$. The Green's functions of the linear nonequilibrium system will be denoted by $G_{0}$. For this linear problem, the result does not depend on $T_{C}$. Finally, the nonlinear interaction $H_{n}$ is turned on, and at time $t=0$, a nonequilibrium steady state is established, see Fig. 1 1 for illustration. The full nonlinear Green's functions will be denoted by $G$.

The density matrices at time $t=-\infty, T$, and $t=0$ are related in the following way in the interaction picture:

$$
\begin{aligned}
\rho(T) & =S_{0}(T,-\infty) \rho(-\infty) S_{0}(-\infty, T), \\
S_{0}\left(t, t^{\prime}\right) & =\mathcal{T} e^{-i \int_{t^{\prime}}^{t} V\left(t^{\prime \prime}\right) d t^{\prime \prime}} \\
\rho(0) & =S(0, T) \rho(T) S(T, 0), \\
S\left(t, t^{\prime}\right) & =\mathcal{T} e^{-i \int_{t^{\prime}}^{t} H_{n}\left(t^{\prime \prime}\right) d t^{\prime \prime}}
\end{aligned}
$$

where $\mathcal{T}$ is the time-order operator.

\section{Equation of motion method}

In this subsection, we derive the equations of motion for Green's functions. The equations of motion can be used to perform systematic perturbation expansion for the Green's functions, or as a starting point for meanfield approximations. There are at least two ways to derive the equations of motion for nonequilibrium Green's functions. The first is to derive the equations of motion for the time-ordered Green's function and then generalize it to the contour-ordered version by evoking the structure isomorphism of the two sets of Green's functions [17]. Another possibility is to consider directly the contour-ordered Green's function 22]. We'll take the latter approach.

We define a general $n$-point contour-ordered Green's function as

$$
\begin{aligned}
& G_{j_{1}, j_{2}, \cdots, j_{n}}^{\alpha_{1}, \alpha_{2}, \cdots, \alpha_{n}}\left(\tau_{1}, \tau_{2}, \cdots, \tau_{n}\right)= \\
& \quad-i\left\langle\mathcal{T}_{\tau} u_{j_{1}}^{\alpha_{1}}\left(\tau_{1}\right) u_{j_{2}}^{\alpha_{2}}\left(\tau_{2}\right) \ldots u_{j_{n}}^{\alpha_{n}}\left(\tau_{n}\right)\right\rangle .
\end{aligned}
$$

The index $\alpha=L, C, R$ labels the region, $j$ labels the degrees of freedom in that region, and $\tau$ is the contour variable. The function is symmetric with respect to simultaneous permutations of the triplet $(\alpha, j, \tau)$. We view 
the function as an analytic function (at least along the path) in variable $\tau$ which varies on the Keldysh contour 23]. We also introduce a generalized $\theta\left(\tau, \tau^{\prime}\right)$ function which is defined to be 1 if $\tau$ is later than $\tau^{\prime}$ along the contour and 0 otherwise. Its derivative is a generalized $\delta$-function, $\delta\left(\tau-\tau^{\prime}\right)=\sigma \delta_{\sigma, \sigma^{\prime}} \delta\left(t-t^{\prime}\right)$. The $\theta$ function can be used to represent the contour-order operator $\mathcal{T}_{\tau}$. For convenience, we allow for $n=0$, which is just a constant $-i$. Our definition is slightly different from that of the usual quantum field theory where our prefactor is always $-i$ (instead of the usual $(-i)^{n / 2}$ ).

To start with, we consider one of the important correlation functions for heat transport, $G^{C, L}$. The first derivative with respect to the second argument always leads to a direct differentiation inside the contour-order operator since the equal-time coordinates $u$ commute,

$$
\frac{\partial G_{j, l}^{C, L}\left(\tau, \tau^{\prime}\right)}{\partial \tau^{\prime}}=-i\left\langle\mathcal{T}_{\tau} u_{j}^{C}(\tau) \dot{u}_{l}^{L}\left(\tau^{\prime}\right)\right\rangle .
$$

The second derivative is similar in this case as $u^{C}$ and $\dot{u}^{L}$ also commute at equal time. Thus, substituting the equation of motion for $u_{L}$ (having identical form in quantum and classical cases), we get

$\frac{\partial^{2} G_{j, l}^{C, L}\left(\tau, \tau^{\prime}\right)}{\partial \tau^{\prime 2}}=-\sum_{m} G_{j, m}^{C, L}\left(\tau, \tau^{\prime}\right) K_{m l}^{L}-\sum_{m} G_{j, m}^{C, C}\left(\tau, \tau^{\prime}\right) V_{m l}^{C L}$.

In matrix notation, it is

$$
\frac{\partial^{2} G^{C, L}\left(\tau, \tau^{\prime}\right)}{\partial \tau^{\prime 2}}+G^{C, L}\left(\tau, \tau^{\prime}\right) K^{L}=-G^{C, C}\left(\tau, \tau^{\prime}\right) V^{C L} .
$$

To solve this differential equation on the contour, we define the (contour-ordered) Green's function of the left lead to satisfy

$$
\frac{\partial^{2} g^{L}\left(\tau^{\prime}, \tau^{\prime \prime}\right)}{\partial \tau^{\prime 2}}+K^{L} g^{L}\left(\tau^{\prime}, \tau^{\prime \prime}\right)=-I \delta\left(\tau^{\prime}-\tau^{\prime \prime}\right) .
$$

We can think of $-g^{L}$ as the inverse of the operator $\partial^{2} / \partial \tau^{\prime 2}+K^{L}$. By multiplying $g^{L}\left(\tau^{\prime}, \tau^{\prime \prime}\right)$ to Eq. (30) from the right, $G^{C, L}\left(\tau, \tau^{\prime}\right)$ to Eq. (31) from the left, and then subtracting the two equations and integrating over the variable $\tau^{\prime}$ along the contour, we get (after integration by part)

$$
\begin{gathered}
G^{C, L}\left(\tau, \tau^{\prime \prime}\right)=\int G^{C, C}\left(\tau, \tau^{\prime}\right) V^{C L} g^{L}\left(\tau^{\prime}, \tau^{\prime \prime}\right) d \tau^{\prime}+ \\
\frac{\partial G^{C, L}\left(\tau, \tau^{\prime}\right)}{\partial \tau^{\prime}} g^{L}\left(\tau^{\prime}, \tau^{\prime \prime}\right)+\left.G^{C, L}\left(\tau, \tau^{\prime}\right) \frac{\partial g^{L}\left(\tau^{\prime}, \tau^{\prime \prime}\right)}{\partial \tau^{\prime}}\right|_{\tau^{\prime}=-\infty+i \epsilon} ^{\tau^{\prime}=-\infty-i \epsilon}
\end{gathered}
$$

The reason that the terms in the second line are identically zero involves some considerations. First, since the central part and left lead are decoupled at the time $\tau=-\infty+i \epsilon$, we must have $G^{C, L}(\tau,-\infty+i \epsilon)=0$ and $\frac{\partial}{\partial \tau^{\prime}} G^{C, L}(\tau,-\infty+i \epsilon)=0$. This takes care of the lower bound. Next, we require that the free left lead Green's function satisfies the boundary condition $g^{L}\left(\tau^{\prime}, \tau^{\prime \prime}\right)=0$, $\frac{\partial}{\partial \tau^{\prime}} g^{L}\left(\tau^{\prime}, \tau^{\prime \prime}\right)=0$, at the upper limit $\tau^{\prime}=-\infty-i \epsilon$. This requirement is equivalent to $g^{\bar{t}}(-\infty)=0$, and $g^{>}(-\infty)=0$, or $g^{r}(-\infty)=0$ (as well as that their first derivatives are zero). The condition $g^{r}(-\infty)=0$ is consistent with the causality requirement of the retarded Green's functions, but the conditions for $g^{\bar{t}}$ or $g^{>}$are insufficient, as the information about temperature of the system is unspecified. We fix the ambiguity by requiring that

$$
g^{>}(t=0)=-i\left\langle u^{L}(0) u^{L}(0)^{T}\right\rangle
$$

be the equilibrium value at inverse temperature $\beta_{L}$. In other words, $g^{L}$ should exactly be the contour-ordered Green's function of the free lead as defined in subsec.IIA. Such a choice is consistent with the adiabatic switch-on. A full justification of the final result,

$$
G^{C, L}\left(\tau, \tau^{\prime \prime}\right)=\int G^{C, C}\left(\tau, \tau^{\prime}\right) V^{C L} g^{L}\left(\tau^{\prime}, \tau^{\prime \prime}\right) d \tau^{\prime},
$$

can also be given from a perturbation expansion, as has been done in Ref. [17].

Equation (34) can be generalized to the case of $G^{C, C, L}$ with a similar result. We now consider the Green's functions of the form $G^{C, C, \cdots, C}$ involving only the central part. The aim is to derive a recursion relation for the central part Green's functions by eliminating references to full Green's functions involving the leads. This type of Green's functions involves two new features, (1) we must take care of the derivatives of the $\theta$ functions which produce commutators $\left[u^{C}, \dot{u}^{C}\right],(2)$ we generate higher order Green's functions due to the nonlinear interactions.

The contour order leads to $n$ ! terms, each of which is a permutation of the displacement variable $u$ from the original order $1,2, \cdots, n$, multiplied by a term of permutation of the arguments $\tau$ from the standard order $\theta\left(\tau_{1}-\tau_{2}\right) \theta\left(\tau_{2}-\tau_{3}\right) \cdots \theta\left(\tau_{n-1}-\tau_{n}\right)$. Differentiation of these $\theta$ functions leads to a sum of contractions between the variable that we are differentiating and all the other variables. This reduces the order of the Green's function by two. We note that

$$
\left[u_{j}^{C}(\tau), \dot{u}_{l}^{C}(\tau)\right]=i \delta_{j l} .
$$

For the moment, we'll consider only the cubic nonlinearity. Four terms are produced when $\ddot{u}^{C}$ is substituted inside the order operator, due to linear coupling $K^{C}$, $V^{C L}, V^{C R}$, and nonlinear $T_{i j k}$. The first three terms are similar in structure to that in Eq. (29). The cubic nonlinear interaction increases the value of $n$ by 1 . We note that the equation for $\ddot{u}^{C}$ is evaluated at the same time $\tau$. This would be complicated if we have to keep track of which $\tau$ 's are equal in the Green's functions. We transfer this information to the coupling $T_{i j k}$ to define the three-body interaction in contour variable form (for the force)

$$
-\sum_{j k} \iint T_{i j k}\left(\tau, \tau^{\prime}, \tau^{\prime \prime}\right) u_{j}^{C}\left(\tau^{\prime}\right) u_{k}^{C}\left(\tau^{\prime \prime}\right) d \tau^{\prime} d \tau^{\prime \prime} .
$$




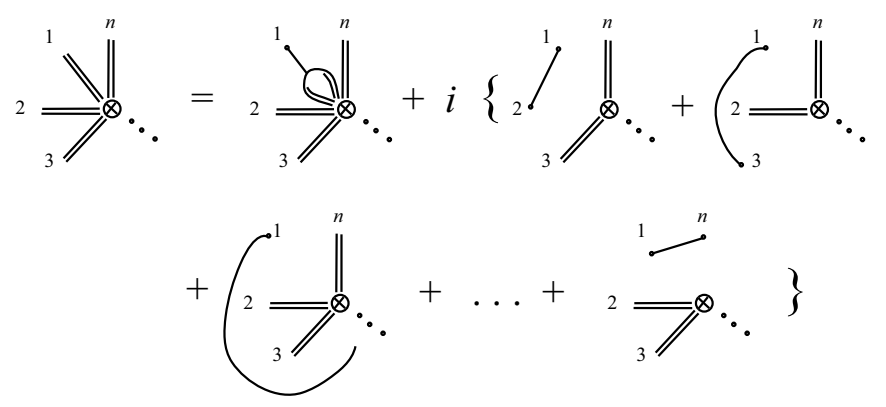

FIG. 2: Recursive expansion rule for Green's functions. A vertex with $n$ double lines denotes an $n$-point Green's function. The single line denotes $G_{0}$. A single-line three-terminal vertex is associated with $T\left(\tau, \tau^{\prime}, \tau^{\prime \prime}\right)$.

Since the contour integral is $\int d \tau=\sum_{\sigma} \sigma \int_{-\infty}^{+\infty} d t$, we must define

$$
T_{i j k}\left(\tau, \tau^{\prime}, \tau^{\prime \prime}\right)=T_{i j k} \sigma^{\prime} \delta_{\sigma, \sigma^{\prime}} \delta\left(t-t^{\prime}\right) \sigma^{\prime \prime} \delta_{\sigma, \sigma^{\prime \prime}} \delta\left(t-t^{\prime \prime}\right) .
$$

This is because our $\delta$-function has the standard property that $\int \delta\left(\tau-\tau^{\prime}\right) d \tau=1$, but when written in the ordinary time $t$ and index $\sigma$, we must have the extra $\sigma$ factor to get the required value of +1 .

The two terms involving the leads produce Green's functions of the form $G^{C, C, \ldots, L}$ and $G^{C, C, \ldots, R}$, which can be replaced by $G^{C, C, \ldots, C}$ using the result Eq. (34) derived earlier. We can now solve for the center-only Green's function using the Green's function of the linear system:

$$
\begin{gathered}
\left(\frac{\partial^{2}}{\partial \tau^{2}}+K^{C}\right) G_{0}\left(\tau, \tau^{\prime}\right)+\int d \tau^{\prime \prime}\left(V^{C L} g^{L}\left(\tau, \tau^{\prime \prime}\right) V^{L C}+\right. \\
\left.V^{C R} g^{R}\left(\tau, \tau^{\prime \prime}\right) V^{R C}\right) G_{0}\left(\tau^{\prime \prime}, \tau^{\prime}\right)=-I \delta\left(\tau-\tau^{\prime}\right) .
\end{gathered}
$$

We then obtain the following simple recursive rules for Green's functions (as shown in Fig. 2):

(1) Replace leg 1 by inserting a nonlinear coupling $T\left(\tau, \tau^{\prime}, \tau^{\prime \prime}\right)$ such that the outer leg is immediately connected with $G_{0}$ while the other two terminals increase the order of the Green's function by 1. Quartic interaction is similar, but the process will increase the order by 2.

(2) Add imaginary unit $i$ times a sum of the $n-1$ graphs formed by pairing each leg with leg 1 and connecting with the propagator $G_{0}$, multiplied by a $(n-2)$ order remaining Green's function.

(3) Symmetrize the graphs, if desired, i.e., do steps (1) and (2) for every leg, $1,2, \cdots, n$, add them up, and then divide by $n$.

The above rules may be conveniently implemented in a symbolic computer language, such as Mathematica.

\section{Feynman diagrams and Dyson equations}

The contour-ordered Green's function can also be obtained by a perturbation expansion of the interaction pic- ture evolution operator (scattering matrix operator) and is expressed as:

$$
\begin{aligned}
G_{j k}\left(\tau, \tau^{\prime}\right) & =-i\left\langle\mathcal{T}_{\tau} u_{j}^{H}(\tau) u_{k}^{H}\left(\tau^{\prime}\right)\right\rangle \\
& =-i\left\langle\mathcal{T}_{\tau} u_{j}^{I}(\tau) u_{k}^{I}\left(\tau^{\prime}\right) e^{-i \int H_{n}^{I}\left(\tau^{\prime \prime}\right) d \tau^{\prime \prime}}\right\rangle_{0},
\end{aligned}
$$

where the displacements refer to the central region; we have dropped the superscript $C$ for brevity. The operators in the top line are in the Heisenberg picture, while that in the second line are in the interaction picture. The Green's function $G_{0}$ of the linear system (when $H_{n}=0$ ) can be computed from that of the free subsystems (an integral equation form of Eq. (38) ):

$$
G_{0}\left(\tau, \tau^{\prime}\right)=g^{C}\left(\tau, \tau^{\prime}\right)+\int d \tau_{1} d \tau_{2} g^{C}\left(\tau, \tau_{1}\right) \Sigma\left(\tau_{1}, \tau_{2}\right) G_{0}\left(\tau_{2}, \tau^{\prime}\right),
$$

where $\Sigma=\Sigma_{L}+\Sigma_{R}$

$$
\Sigma_{L}=V^{C L} g^{L} V^{L C},
$$

similarly for $\Sigma_{R}$. This Dyson equation can be derived by considering the first step of the adiabatic switching-on process. Since it is a linear system, the self-energy $\Sigma$ is known exactly.

By expanding the exponential in Eq. (39), a series in the nonlinear interaction strength is obtained. The reduction in terms of the unperturbed Green's function $G_{0}$ relies on the fact that Wick's theorem 24] is applicable here. Although a formal proof of Wick's theorem is difficult, we can see that the density matrix $\rho(0)$ must be quadratic in exponential. This is because the $\rho(-\infty)$ is quadratic and the system is linear. The fact that the equation of motion method and the perturbation expansion give identical results is a confirmation of the validity of Wick's theorem.

The expansion contains connected as well as disconnected Feynman diagrams. The disconnected diagrams are constant in time, and give rise to a thermal expansion effect. We can show that these diagrams do not contribute to the thermal transport, as they are proportional to $\delta(\omega)$ in the frequency domain. The thermal current formula has a factor of $\omega$ which makes it zero.

Finally, the connected part of the Green's function satisfies a similar contour-ordered Dyson equation relating $G_{c}$ to $G_{0}$ through a nonlinear self-energy $\Sigma_{n}$ :

$G_{c}\left(\tau, \tau^{\prime}\right)=G_{0}\left(\tau, \tau^{\prime}\right)+\int d \tau_{1} d \tau_{2} G_{0}\left(\tau, \tau_{1}\right) \Sigma_{n}\left(\tau_{1}, \tau_{2}\right) G_{c}\left(\tau_{2}, \tau^{\prime}\right)$.

In ordinary Green's functions and in frequency domain ( $\omega$ argument suppressed), the Dyson equations have solutions [18]:

$$
\begin{aligned}
G_{0}^{r} & =G_{0}^{a \dagger}=\left((\omega+i \eta)^{2} I-K^{C}-\Sigma^{r}\right)^{-1}, \\
G_{0}^{<} & =G_{0}^{r} \Sigma^{<} G_{0}^{a}, \\
G_{c}^{r} & =\left(G_{0}^{r-1}-\Sigma_{n}^{r}\right)^{-1}, \\
G_{c}^{<} & =G_{c}^{r}\left(\Sigma^{<}+\Sigma_{n}^{<}\right) G_{c}^{a} .
\end{aligned}
$$




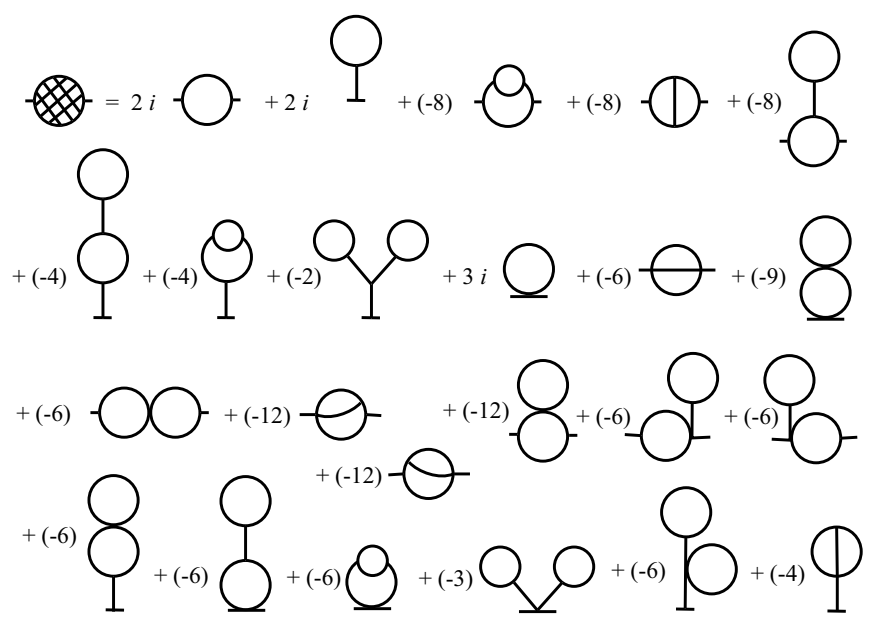

FIG. 3: The Feynman diagrams for nonlinear self-energy $\Sigma_{n}$ with the prefactors for graphs of cubic and quartic interactions to second order in $\hbar$.

The connected part of the Green's function $G_{c}$ will be used for $G$ in Eq. (52) below, and the extra subscript $c$ will be dropped from this point.

In Fig. 3 we give the Feynman diagrams to "second order" of the nonlinear interactions for cubic and quartic terms, where the graphs have explicitly $\hbar$ and $\hbar^{2}$ in the coefficients; the neglected graphs have higher powers of $\hbar$. These diagrams are the same as those in Ref. [25], but the interpretation is different. It is for the contour-ordered Green's functions here and for the retarded Green's functions of the creation/annihilation operators in Ref. 25]. To find the actual expressions for each of the diagrams, we have the following simple rules: (1) label each interaction vetex line by the $\tau$ variables and site indices, with the interaction function $T_{i j k}\left(\tau_{i}, \tau_{j}, \tau_{k}\right)$ (or $T_{i j k l}\left(\tau_{i}, \tau_{j}, \tau_{k}, \tau_{l}\right.$ ) for the quartic interaction). (2) Each line connecting the labels is associated with a propagator $G_{0}$. (3) Integrate over all the internal variables along the contour; sum over the internal site indices. Most of the integrations are easy, as $T_{i j k}(\cdots)$ contains two $\delta$-functions in $\tau$. For example, the second graph is

$$
\begin{aligned}
& \sum_{j_{3}, j_{4}, j_{5}, j_{6}} \int \int \\
& G_{0, j_{3} j_{4}}\left(\tau_{3}, \tau_{4}\right) T_{j_{4}, j_{5}, j_{6}}\left(\tau_{4}, \tau_{5}, \tau_{6}\right) G_{0, j_{5} j_{6}}\left(\tau_{5}, \tau_{6}\right),
\end{aligned}
$$

where $\tau_{1}$ and $\tau_{2}$ are external variables and the rest are internal. After integrations, we get

$\sum_{j_{3}, j_{4}, j_{5}, j_{6}} \int d \tau_{4} T_{j_{1}, j_{2}, j_{3}} \delta\left(\tau_{1}-\tau_{2}\right) G_{0, j_{3} j_{4}}\left(\tau_{1}, \tau_{4}\right) T_{j_{4}, j_{5}, j_{6}} G_{0, j_{5} j_{6}}\left(\tau_{4}, \tau_{4}\right)$.

Now we can rewrite in real time $t$ and the branch in$\operatorname{dex} \sigma$ using the mapping $G\left(\tau, \tau^{\prime}\right) \rightarrow G^{\sigma \sigma^{\prime}}\left(t, t^{\prime}\right), \int d \tau \rightarrow$ $\sum_{\sigma= \pm 1} \int_{-\infty}^{\infty} \sigma d t$, and $\delta\left(\tau-\tau^{\prime}\right) \rightarrow \sigma \delta_{\sigma, \sigma^{\prime}} \delta\left(t-t^{\prime}\right)$. Noting that $G_{0}\left(\tau_{4}, \tau_{4}\right)=G_{0}(0)$ by time translational invariance, we have

$$
\begin{aligned}
\sigma_{1} \delta_{\sigma_{1}, \sigma_{2}} \delta\left(t_{1}-t_{2}\right) & \sum_{j_{3}, j_{4}, j_{5}, j_{6}, \sigma_{4}} T_{j_{1}, j_{2}, j_{3}} T_{j_{4}, j_{5}, j_{6}} \\
& \int G_{0, j_{3}, j_{4}}^{\sigma_{1}, \sigma_{4}}\left(t_{1}-t_{4}\right) G_{0, j_{5}, j_{6}}^{\sigma_{4}, \sigma_{4}}(0) \sigma_{4} d t_{4} .
\end{aligned}
$$

The result can now be transformed to frequency domain if desired.

\section{E. Thermal current and conductance}

We define the current flow from the left lead to the central region as

$$
I_{L}=-\left\langle\dot{H}_{L}(t)\right\rangle
$$

The interpretation of the current is somewhat subtle as the leads are semi-infinite in extent. The definition is meaningful. By the Heisenberg equation of motion, we obtain, at $t=0, I_{L}=\left\langle\left(\dot{u}^{L}\right)^{T} V^{L C} u^{C}\right\rangle$. The expectation value can be expressed in terms of a Green's function $G_{C L}^{<}\left(t, t^{\prime}\right)=-i\left\langle u^{L}\left(t^{\prime}\right) u^{C}(t)^{T}\right\rangle^{T}$. Using the fact that operators $u$ and $\dot{u}$ are related in Fourier space as $\dot{u}[\omega]=(-i \omega) u[\omega]$, we can eliminate the derivative and get,

$$
I_{L}=-\frac{1}{2 \pi} \int_{-\infty}^{\infty} \operatorname{Tr}\left(V^{L C} G_{C L}^{<}[\omega]\right) \omega d \omega
$$

Using the result derived earlier relating the mixed leadcenter Green's function to the center-only Green's function and applying the Langreth theorem [18, 26] to write contour-ordered Green's function in ordinary Green's functions in frequency domain, we have $G_{C L}^{<}[\omega]=$ $G_{C C}^{r}[\omega] V^{C L} g_{L}^{<}[\omega]+G_{C C}^{<}[\omega] V^{C L} g_{L}^{a}[\omega]$. The final expression for the energy current is

$$
I_{L}=-\frac{1}{2 \pi} \int_{-\infty}^{+\infty} d \omega \omega \operatorname{Tr}\left(G^{r}[\omega] \Sigma_{L}^{<}[\omega]+G^{<}[\omega] \Sigma_{L}^{a}[\omega]\right)
$$

where the self-energy due to the interaction with the lead is $\Sigma_{L}=V^{C L} g_{L} V^{L C}$. For simplicity, we have dropped the subscript $C$ on the Green's functions denoting the central region. This result is the phonon analog of the electronic current formula [17, 18].

Since the energy current must be conserved, we have $I_{L}=-I_{R}$. In addition, $I_{L}$ is real. We can obtain a symmetrized expression for the current

$$
\begin{aligned}
I= & \frac{1}{4}\left(I_{L}+I_{L}^{*}-I_{R}-I_{R}^{*}\right)=\frac{1}{4 \pi} \int_{0}^{\infty} d \omega \\
& \omega \operatorname{Tr}\left\{\left(G^{r}-G^{a}\right)\left(\Sigma_{R}^{<}-\Sigma_{L}^{<}\right)+i G^{<}\left(\Gamma_{R}-\Gamma_{L}\right)\right\},
\end{aligned}
$$

where $\Gamma_{\alpha}=i\left(\Sigma_{\alpha}^{r}-\Sigma_{\alpha}^{a}\right)$.

We define the thermal conductance as

$$
\bar{\kappa}=\lim _{\Delta T \rightarrow 0} \frac{I}{\Delta T}=\frac{\delta I}{\delta T},
$$




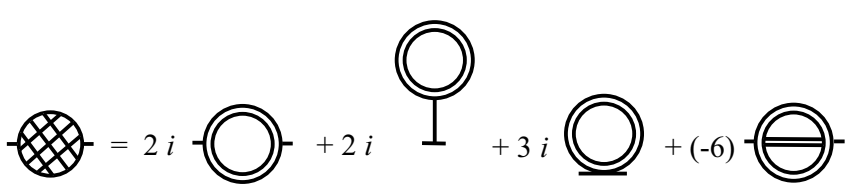

FIG. 4: Mean-field approximation to the self-energy. The double line denotes the full Green's function $G$; the single line is for $G_{0}$.

where $\Delta T$ is the difference of the temperatures in leads, such that $T_{L}=T+\Delta T / 2$ and $T_{R}=T-\Delta T / 2$. Using Eq. (53), we can actually take the limit $\Delta T \rightarrow 0$ by introducing variational derivatives of the Green's functions, self-energies, or current as, e.g.,

$$
\frac{\delta G}{\delta T}=\lim _{\Delta T \rightarrow 0} \frac{G\left(T_{L}, T_{R}\right)-G(T, T)}{T_{L}-T_{R}} .
$$

The difference in the numerator is the value of a function when the leads are at two different temperatures $T_{L}$ and $T_{R}$, respectively, minus the value when the system is in equilibrium at $T_{L}=T_{R}=T$. With this notation, we can express the conductance in a form similar to the Landauer formula [27] for the ballistic transport as

$$
\bar{\kappa}=\frac{1}{2 \pi} \int_{0}^{\infty} d \omega \omega \tilde{T}[\omega] \frac{\partial f(\omega)}{\partial T}
$$

with an effective transmission coefficient,

$$
\begin{aligned}
\tilde{T}[\omega]= & \frac{1}{2} \operatorname{Tr}\left\{G^{r}\left(\Gamma_{L}+\frac{1}{2} \Gamma_{n}-S\right) G^{a} \Gamma_{R}\right\}+ \\
& \frac{1}{2} \operatorname{Tr}\left\{G^{a} \Gamma_{L} G^{r}\left(\Gamma_{R}+\frac{1}{2} \Gamma_{n}+S\right)\right\},
\end{aligned}
$$

where the nonlinear effect is reflected in the extra terms, $\Gamma_{n}=i\left(\Sigma_{n}^{r}-\Sigma_{n}^{a}\right)$, and

$$
S=i\left[f\left(\frac{\delta \Sigma_{n}^{r}}{\delta T}-\frac{\delta \Sigma_{n}^{a}}{\delta T}\right)-\frac{\delta \Sigma_{n}^{<}}{\delta T}\right]\left(\frac{\partial f}{\partial T}\right)^{-1} .
$$

Equation (57) is a generalization of the Caroli formula [28] for ballistic transport. We'll discuss in Sec. IIIB how the variational derivatives of the self-energy can be computed.

\section{F. Mean-field approximation}

We have seen in Ref. 15] that high-order Feynman diagrams are important at high temperatures. However, it is computationally very expensive to include these highorder diagrams, although we can do calculations on very small systems such as a three-site model. The standard technique is to use a mean-field approximation which in some sense partially takes into account these high-order graphs. We propose an approximation to the self-energy shown in Fig. 4, where the double line represents the nonlinear full Green's function $G$, while a single line is the usual bare Green's function $G_{0}$. With such a choice, we find that to next order, all the cubic interaction graphs are reproduced exactly (including the prefactor), except that two of the diagrams are missing.

With the self-consistent mean-field approximation, the solution has to be found iteratively. The convergence of the iterative process becomes an issue. With only cubic interactions, the system of equations will not converge at high temperatures. We understand that this is simply a consequence of the unstable nature when only cubic terms are used. We thus add the fourth order potential which is essential for stability. However, the convergence is still difficult at high temperatures, mainly due to the singular nature of the Bose distribution.

\section{G. Classical limit, molecular dynamics}

At high temperatures, the classical molecular dynamics (MD) is a correct and numerically exact method for the heat conduction problem. However, the standard heat baths used in MD such as Nosé-Hoover do not correspond to exactly the action of the leads in our models. A correct heat bath should follow a Langevin dynamics with colored noises. Dhar [29] has given a derivation for the junction problem. The idea is to solve the lead degrees of freedom $u^{L}$ and $u^{R}$ formally first, which is easy since it is a linear system. The result is then substituted into the equation for the center region. This gives the following equation:

$\ddot{u}^{C}=-K^{C} u^{C}+F_{n}\left(u^{C}\right)-\int_{T}^{t} \Sigma^{r}\left(t, t^{\prime}\right) u^{C}\left(t^{\prime}\right) d t^{\prime}+\xi_{L}+\xi_{R}$,

where $F_{n}$ is the nonlinear force, $\Sigma^{r}$ is the same retarded self-energy of the lead, $\Sigma^{r}=\Sigma_{L}^{r}+\Sigma_{R}^{r}$, used in the nonequilibrium Green's function calculation, but in the time domain. By integration by part, we could also cast Eq. (59) in a standard generalized Langevin form [30] involving a damping force, but $K^{C}$ has to be shifted to $K^{C}+\Sigma^{r}[\omega=0]$. An extra contribution from the left lead is

$$
\xi_{L}(t)=V^{C L}\left(g_{L}^{r}(t, T) \dot{u}^{L}(T)-\frac{\partial g_{L}^{r}(t, T)}{\partial T} u^{L}(T)\right),
$$

where $g_{L}^{r}$ is the retarded Green's function of the left lead in the time domain. The expression for the right lead $\xi_{R}$ is similar. A time $T$ in the lower limit in the integral and Eq. (60) is some time in the remote past where the system is decoupled and the leads are in respective thermal equilibrium. We'll take the limit $T \rightarrow-\infty$. We turn Eq. (59) into a stochastic differential equation by requiring that $u^{L}(T)$ and $\dot{u}^{L}(T)$ are random variables satisfying the Boltzmann-Gibbs distribution

$$
P\left(u^{L}, \dot{u}^{L}\right) \propto e^{-\beta_{L}\left(\frac{1}{2}\left(\dot{u}^{L}\right)^{T} \dot{u}^{L}+\frac{1}{2}\left(u^{L}\right)^{T} K^{L} u^{L}\right)} .
$$


Thus, $\xi_{L}$ is colored noise with the properties

$$
\begin{aligned}
\left\langle\xi_{L}(t)\right\rangle= & 0 \\
\beta_{L}\left\langle\xi_{L}(t) \xi_{L}\left(t^{\prime}\right)^{T}\right\rangle= & V^{C L}\left(\dot{g}_{L}^{r}(t, T) \frac{1}{K^{L}} \dot{g}_{L}^{r}\left(t^{\prime}, T\right)^{T}\right. \\
& \left.+g_{L}^{r}(t, T) g_{L}^{r}\left(t^{\prime}, T\right)^{T}\right) V^{L C} \\
=- & \int_{t}^{\infty} \Sigma_{L}^{r}\left(t^{\prime \prime}, t^{\prime}\right) d t^{\prime \prime}, \quad\left(t>t^{\prime}\right) .
\end{aligned}
$$

The last line of the above equation is a kind of "fluctuation-dissipation" theorem. We note that the dot in $g$ denotes the derivative with respect to $T$. For a sensible heat bath, the noise should not depend on $T$, but only on the difference $t-t^{\prime}$. Indeed, this can be done if we rewrite the result in vibrational eigen-modes. We can write the Fourier transform of the noise correlation as

$$
\operatorname{Im} 2 V^{C L}\left(\left(K^{L}\right)^{-1 / 2} \frac{1}{\left(\omega^{2}-i \eta\right) I-K^{L}}\right) V^{L C},
$$

where the square root of a matrix is defined by its eigenvalue decomposition taking only the positive eigen frequencies. This is well defined since $K^{L}$ is positive definite. The properties of the right lead noise $\xi_{R}$ are analogous. Such a set of stochastic differential equations can be simulated on a computer.

\section{IMPLEMENTATION DETAILS}

\section{A. Surface Green's functions}

The Green's functions of the free leads $g^{r}$ are required in calculation for the lead self energies $\Sigma$. Although the lead Green's function is a semi-infinite matrix, due to a localized coupling $V^{L C}$ or $V^{C R}$, only a corner set of elements is required. These submatrix elements are termed surface Green's functions. The surface Green's function can be computed rather quickly by recursive iterations 31] or decimation. For completeness, we give an algorithm in pseudo-code form. The reader is referred to the literature for the derivation of such algorithm.

The program takes three square matrices of equal size for $k_{00}, k_{11}$, and $k_{01}$. The matrix $k_{00}$ is the block immediately adjacent to the center, while $k_{11}$, and $k_{01}=k_{10}^{T}$ are repeated for the semi-infinite chain of the lead. They form the whole dynamic matrix of the lead in the following way:

$$
K^{R}=\left(\begin{array}{cccc}
k_{00} & k_{01} & 0 & \cdots \\
k_{10} & k_{11} & k_{01} & 0 \\
0 & k_{10} & k_{11} & k_{01} \\
\cdots & 0 & k_{10} & \ddots
\end{array}\right)
$$

The left arrow ' $\leftarrow$ ' below denotes assignment; $\epsilon$ is an error tolerance.

$$
\begin{aligned}
s & \leftarrow k_{00} \\
e & \leftarrow k_{11} \\
\alpha & \leftarrow k_{01} \\
\beta & \leftarrow \alpha^{T} \\
g & \leftarrow\left[(\omega+i \eta)^{2} I-e\right]^{-1} \\
\text { do } & s^{\prime} \leftarrow s+\alpha g \beta \\
& \text { if }\left|s^{\prime}-s\right|<\epsilon \text { exit } \\
& e^{\prime} \leftarrow e+\alpha g \beta+\beta g \alpha \\
& \alpha^{\prime} \leftarrow \alpha g \alpha \\
& \beta^{\prime} \leftarrow \beta g \beta \\
& s \leftarrow s^{\prime} \\
& e \leftarrow e^{\prime} \\
& \alpha \leftarrow \alpha^{\prime} \\
& \beta \leftarrow \beta^{\prime} \\
& g \leftarrow\left[(\omega+i \eta)^{2} I-e\right]^{-1} \\
\text { end do } & \\
g^{r} & \leftarrow\left[(\omega+i \eta)^{2} I-s\right]^{-1} .
\end{aligned}
$$

\section{B. Fast Fourier transform and variational derivatives}

The diagrams in the mean-field approximation can be computed in the time domain easily and efficiently; the convolutions in frequency domain become simple multiplications in time domain. Thus we have adapted a fast Fourier transform method for the calculation. This reduces the computational complexity, particularly for the quartic interaction terms. Typically $10^{3}$ Fourier grid points are needed for good convergence. Using the Message Passing Interface (MPI), a parallel implementation with data partition is made. Good linear speed-up is obtained upto 64 processes.

In the mean-field treatment of the heat transport, there is a need to find the variational derivatives of the Green's functions and self-energy for the conductance calculation. This is done by solving iteratively the set of equations

$$
\begin{aligned}
\delta G^{r} & =G^{r} \delta \Sigma_{n}^{r} G^{r}, \\
\delta G^{<} & =G^{r} \delta \Sigma_{n}^{r} G^{<}+G^{r}\left(\delta \Sigma^{<}+\delta \Sigma_{n}^{<}\right) G^{a}+G^{<} \delta \Sigma_{n}^{a} G^{a}
\end{aligned}
$$

The expressions for $\delta \Sigma_{n}^{r}$ and $\delta \Sigma_{n}^{<}$can be obtained by differentiating the self-energy results directly. For convergence control, linear mixing of the new and old values is used.

\section{APPLICATIONS}

\section{A. One-dimensional cubic onsite model}

One important question to ask about the nonequilibrium formulation is that whether the theory gives ballistic and diffusive heat transport in a single framework. Clearly, if the nonlinear self-energy $\Sigma_{n}$ can be computed accurately, there is no doubt that the answer is yes. However, this is not clear if only the leading order perturbation theory is used. In Ref. [32], Spohn gives a derivation 
of a Boltzmann equation for the phonons in a simple 1D chain with onsite cubic nonlinearity. It is clear that in the Boltzmann equation framework, we obtain Fourier's law and diffusive heat transport. We demonstrate in this subsection that diffusive heat transport is not observed if only perturbative result is used. This subsection also serves as an example of illustration of the concepts discussed earlier by a simple and concrete model.

We consider a $1 \mathrm{D}$ chain with inter-particle spring constant $K$ and onsite spring constant $K_{0}$. We take a simple cubic onsite potential for the interaction, $\frac{1}{3} t \sum_{j} u_{j}^{3}$, for $j$ belonging to the central region. This means that $T_{i j k}=t \delta_{i j} \delta_{i k}$. Apart from the nonlinear interaction, the left lead, central region, and the right lead are identical. The classical equation of motion is

$$
\ddot{u}_{j}=K u_{j-1}+\left(-2 K-K_{0}\right) u_{j}+K u_{j+1}-t_{j} u_{j}^{2},
$$

where $t_{j}=t$ for $1 \leq j \leq N$ and $t_{j}=0$ for $j<1$ or $j>N$.

The retarded Green's function $G_{0}^{r}$ can be obtained by solving the linear equation $\left[(\omega+i \eta)^{2}-\tilde{K}\right] G_{0}^{r}=I$, where (infinite in both directions) matrix $\tilde{K}$ is $2 K+K_{0}$ on the diagonal and $-K$ on the first off-diagonals. The explicit solution is

$$
G_{0 j l}^{r}[\omega]=\frac{\lambda_{1}^{|j-l|}}{\left(\lambda_{1}-\lambda_{2}\right) K},
$$

where $\lambda_{1}$ and $\lambda_{2}$ are the roots of the equation

$$
K \lambda^{-1}+\Omega+K \lambda=0, \quad \Omega=(\omega+i \eta)^{2}-2 K-K_{0} .
$$

Note that $\lambda_{1} \lambda_{2}=1$. We define the two roots such that $\left|\lambda_{1}\right|<1$ and $\left|\lambda_{2}\right|>1$.

The surface Green's function $g^{r}$ satisfies a similar equation as $G_{0}^{r}$ except that it is semi-infinite in extent. We consider the left lead $(j \leq 0)$. The result for the right lead is identical. Since the matrix $V^{L C}$ is nonzero only for one corner element, we need the $g_{0}=g_{00}$ component of the matrix $g^{r}$. Consider only the $j=0$ column, the equations for $g^{r}$ in component form are

$$
\begin{gathered}
\Omega g_{0}+K g_{-1}=1 \\
K g_{j-1}+\Omega g_{j}+K g_{j+1}=0, \quad j=-1,-2, \cdots
\end{gathered}
$$

Substituting the trial solution $g_{j}=c \lambda_{1}^{-j}$, we find that

$$
g_{0}=-\frac{\lambda_{1}}{K} .
$$

The "less than" Green's function for the left lead is then (c.f. Eq. (16))

$$
g_{L}^{<}=2 i f_{L} \operatorname{Im} g_{0}=-2 i f_{L} \frac{\operatorname{Im} \lambda_{1}}{K},
$$

where $f_{L}$ is the Bose-Einstein distribution function at the temperature of the left lead. The $G_{0}^{<}$Green's function can be obtained with the equation $G_{0}^{<}=G_{0}^{r} \Sigma^{<} G_{0}^{a}$,
$\Sigma=\Sigma_{L}+\Sigma_{R} ; \Sigma_{L}=V^{C L} g_{L} V^{L C}$, similarly for $\Sigma_{R}$. The matrix elements of $\Sigma^{<}$are all zero except that $\Sigma_{11}=K^{2} g_{L}^{<}$and $\Sigma_{N N}=K^{2} g_{R}^{<}$. Using these results, after some calculation, we can write

$$
G_{0 j l}^{<}[\omega]=\frac{f_{L} \lambda_{1}^{j-l}+f_{R} \lambda_{1}^{l-j}}{\left(\lambda_{1}-\lambda_{2}\right) K} \Theta(\omega) .
$$

The $\Theta(\omega)$ function is defined to be 1 inside the phonon band and zero outside the band; more precisely, $\Theta(\omega)=1$ if $K_{0}<\omega^{2}<4 K+K_{0}$, and is 0 otherwise.

Due to the onsite cubic nonlinearity, the leading order nonlinear self-energy calculation is simple. The first graph contribution is

$$
{ }^{1} \Sigma_{n_{j l}}^{\sigma \sigma^{\prime}}(t)=2 i t^{2}\left[G_{0 j l}^{\sigma \sigma^{\prime}}(t)\right]^{2} .
$$

The contribution from the second graph is a constant in $\omega:$

$$
{ }^{2} \Sigma_{n j l}^{\sigma \sigma^{\prime}}[\omega]=2 i t^{2} \sigma \delta_{\sigma, \sigma^{\prime}} \delta_{j l} \sum_{\sigma^{\prime \prime}, s} \sigma^{\prime \prime} G_{0 j s}^{\sigma \sigma^{\prime \prime}}[0] G_{0 s s}^{\sigma_{s s}^{\prime \prime} \sigma^{\prime \prime}}(0) .
$$

Both the summation over $\sigma^{\prime \prime}$ and over index $s$ can be performed analytically. We find

$$
{ }^{2} \Sigma_{n j l}^{\sigma \sigma^{\prime}}[\omega]=2 i t^{2} \sigma \delta_{\sigma, \sigma^{\prime}} \delta_{j l} \bar{G}_{j}^{r}[0] \bar{G}^{t}(0),
$$

where

$$
\begin{aligned}
\bar{G}_{j}^{r}[\omega] & =\frac{1+\lambda_{1}-\lambda_{1}^{j}-\lambda_{1}^{N-j+1}}{\left(1-\lambda_{1}\right)\left(\lambda_{1}-\lambda_{2}\right) K}, \\
\bar{G}^{t}[\omega] & =\frac{1+\left(f_{L}+f_{R}\right) \Theta(\omega)}{\left(\lambda_{1}-\lambda_{2}\right) K}, \\
\bar{G}^{t}(0) & =\frac{1}{2 \pi} \int_{-\infty}^{+\infty} \bar{G}^{t}[\omega] d \omega .
\end{aligned}
$$

For numerical accuracy, we compute the conductance directly instead of using finite differences. Let the temperature on the left lead be $T_{L}=T+\Delta T / 2$ and right lead be $T_{R}=T-\Delta T / 2$. We define the thermal conductance as that in Eq. (54) and the effective transmission by Eq. (56). For the expression for effective transmission $\tilde{T}[\omega]$, we also need to take the limit $\Delta T \rightarrow 0$. The advantage of doing this is that the leading terms corresponding to equilibrium Green's functions cancel exactly, since there should be no heat current when $T_{L}=T_{R}$. We can also equivalently define the temperature variation through the expansion:

$$
G^{r}=G_{\mathrm{eq}}^{r}+\frac{\delta G^{r}}{\delta T} \Delta T+O\left(\Delta T^{2}\right),
$$

and similarly for $G^{<}$. We can simplify the effective transmission as

$$
\begin{aligned}
& \tilde{T}[\omega]= \frac{1}{2} \operatorname{Tr}\left\{\left(G^{r}-G^{a}\right)\left(\Sigma_{R}^{<}-\Sigma_{L}^{<}\right)+\right. \\
&\left.i G^{<}\left(\Gamma_{R}-\Gamma_{L}\right)\right\} /\left(f_{L}-f_{R}\right) \\
&=i K\left(\operatorname{Im} \lambda_{1}\right)\left(F_{11}^{L}-F_{N N}^{R}\right),
\end{aligned}
$$




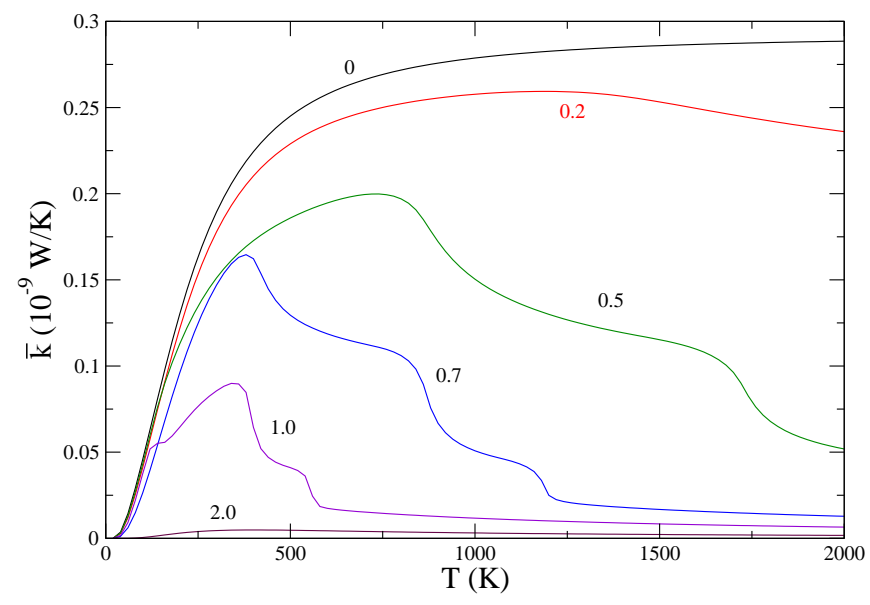

FIG. 5: Thermal conductance of the cubic onsite model as a function of temperature for several values of the cubic coupling strength $t=0,0.2,0.5,0.7,1.0$, and $2.0 \mathrm{eV} /\left(\AA^{3} \mathrm{u}^{3 / 2}\right)$. The length of center chain is $N=5$.

where the $F$ function is defined as

$$
F^{L}=\frac{1}{2}\left(G^{r}-G^{a}\right)+\left[f \frac{\delta\left(G^{r}-G^{a}\right)}{\delta T}-\frac{\delta G^{<}}{\delta T}\right]\left(\frac{\partial f}{\partial T}\right)^{-1} .
$$

$F^{R}$ is the same except that the first term is negative. In evaluating the $F$ functions, we should set $T_{L}=T_{R}=$ $T$ as we have already taken the $\Delta T \rightarrow 0$ limit. The variations required in Eq. (85) can be computed by the variation in nonlinear self-energy, which in turn can be computed by the variation in the linear Green's function $G_{0}$. Due to the Dyson equation, the variation of the retarded Green's function has a simple result:

$$
\frac{\delta G^{r}}{\delta T}=G^{r} \frac{\delta \Sigma_{n}^{r}}{\delta T} G^{r}
$$

The variation in $G^{<}$is rather complicated:

$$
\begin{aligned}
\delta G^{<}= & \frac{\delta G^{<}}{\delta T} \Delta T=G^{r}\left\{\Sigma_{n}^{r} \delta G_{0}^{<}+\right. \\
& \left.\delta \Sigma_{n}^{r}\left[\bar{G}_{0}^{<}+G^{r}\left(\Sigma_{n}^{<} G^{a}+\Sigma_{n}^{r} \bar{G}_{0}^{<}\right)\right]\right\}- \text {h.c. } \\
& +\delta G_{0}^{<}+G^{r}\left(\delta \Sigma_{n}^{<}+\Sigma_{n}^{r} \delta G_{0}^{<} \Sigma_{n}^{a}\right) G^{a},
\end{aligned}
$$

where $\bar{G}_{0}^{<}=G_{0}^{<}\left(I+\Sigma_{n}^{a} G^{a}\right)$, h.c. stands for the Hermitian conjugate of the preceding term, and $\delta G_{0}^{r}=0$,

$$
\begin{aligned}
\frac{\delta G_{0 j l}^{<}}{\delta T} & =\frac{\lambda_{1}^{j-l}-\lambda_{1}^{l-j}}{2\left(\lambda_{1}-\lambda_{2}\right) K} \Theta(\omega) \frac{\partial f}{\partial T}, \\
\delta \Sigma_{n_{j l}}^{r,<}[\omega] & \left.=\frac{4 i t^{2}}{2 \pi} \int_{-\infty}^{+\infty} d \omega^{\prime} G_{0 j l}^{r,<}\left[\omega-\omega^{\prime}\right] \delta G_{0 j l}<\omega^{\prime}\right] .
\end{aligned}
$$

We have made analytic calculations as far as possible. The rest of integrations and matrix inversions are calculated numerically. We use physical units with $K=0.625 \mathrm{eV} /\left(\AA^{2} \mathrm{u}\right), K_{0}=0.1 K$, (u for unified atomic mass units). Figure 5 shows the temperature dependence

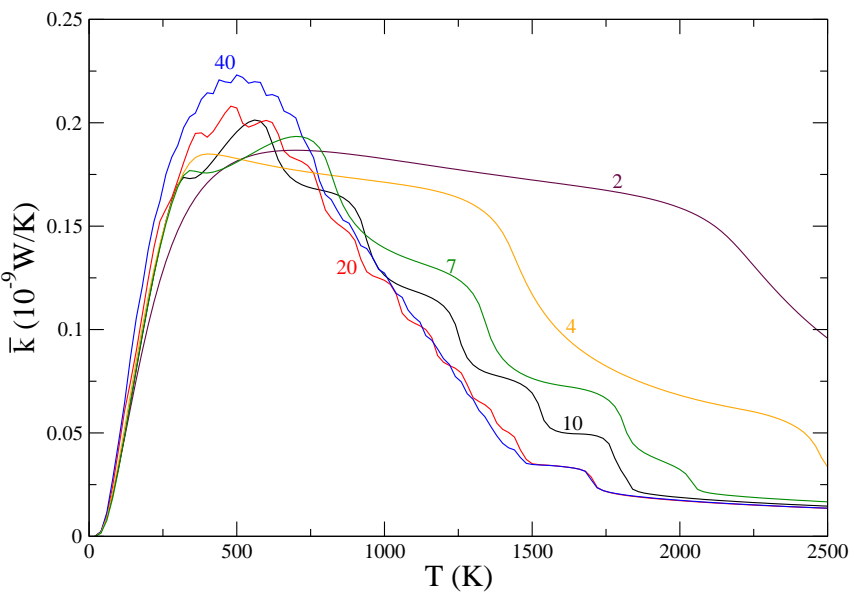

FIG. 6: Thermal conductance as a function of temperature for several system lengths $N$ with $t=0.5 \mathrm{eV} /\left(\AA^{3} \mathrm{u}^{3 / 2}\right)$.

of the thermal conductance $\bar{\kappa}$ of a short nonlinear chain of length $N=5$. The $t=0$ curve is the ballistic Landauer formula result, the rest of the curves show the effect of nonlinearity. The prominent feature here is that the conductance decreases quickly as the nonlinear coupling $t$ increases from 0 to 2 . In particular, the system behaves like a thermal insulator if the on-site nonlinearity is large enough. The thermal conductance is small at low temperatures and at high temperatures, as was found experimentally. At the extremely high temperatures, the conductance decreases with temperature according to $1 / T$ (data not shown). This agrees with several other theories of thermal conductance at high temperatures.

Can we see diffusive transport in this model (under the perturbative approximation)? The answer is no. In Fig. 6, we show the size dependence for a fixed $t$. At temperatures below $200 \mathrm{~K}$, the results are essentially independent of the lengths, showing ballistic heat transport. At around $2000 \mathrm{~K}$, from sizes 2 to 10 , we see decrease of the thermal conductance roughly as $1 / N$, but this trend does not continue, and the conductance saturates to some value for systems larger than 20. This feature is generic; the FPU model with first-order perturbation treatment also has a similar behavior. The curves also display some oscillatory structure. The number of oscillations appears to be roughly equal to half of the chain length $N$. The feature is damped out as the chain becomes longer. It would be nice if a molecular dynamics simulation could confirm these features.

The failure to see diffusive behavior may be understandable as our result takes into account only the firstorder perturbation in the nonlinear interaction. Other features seem qualitatively correct in comparison with real systems; this is very encouraging. 


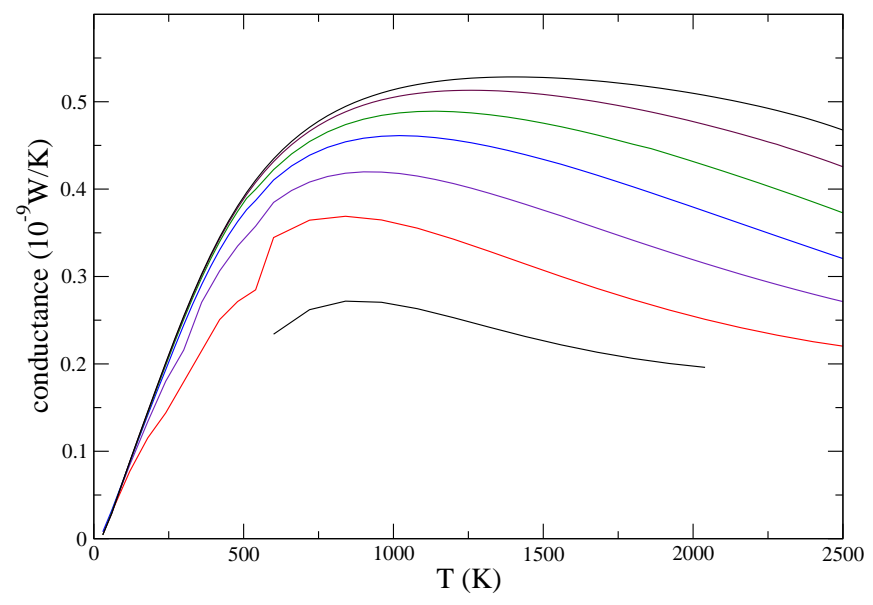

FIG. 7: Thermal conductance of a Fermi-Pasta-Ulam chain with chain lengths $4,8,16,32,64,128,256$, from top to bottom. Semi-infinite one-dimensional harmonic chains serve as heat baths with spring constants $k_{L}=2.31$ and $k_{R}=2.25$ $\mathrm{eV} /\left(\mathrm{u}^{2}\right)$ for the left and right leads, respectively. Small onsite quadratic potentials are applied with $k_{L}^{\text {onsite }}=0.006$ and $k_{R}^{\text {onsite }}=0.01 \mathrm{eV} /\left(\mathrm{u} \AA^{2}\right)$.

\section{B. Fermi-Pasta-Ulam model}

The Fermi-Pasta-Ulam (FPU) model has been studied as a prototype model in nonlinear dynamics 33. In recent years, a lot of works have been done on the thermal transport in the FPU model [34, 35]. By molecular dynamics and mode-coupling theory, Lepri et al. [36, 37] found that the thermal transport in such a system does not have an ordinary diffusive behavior in the sense that the thermal conductivity diverges with the system size as some power, $L^{\alpha}$. The actual value of the exponent $\alpha$ is somewhat controversial. The earlier studies gave $2 / 5$, but the latest analysis suggests $1 / 3$ [38, 39].

Quantum thermal transport in FPU model has seldom been studied. In particular, the change from ballistic to diffusive transport has not been addressed. This question can not be correctly answered within molecular dynamics approach as the ballistic transport is quantummechanical in nature.

We use realistic model parameters derived from expanding the Morse potential, $V(x)=D\left(e^{-a x}-1\right)^{2}$ with $D=3.8 \mathrm{eV}, a=1.88 \AA^{-1}$, up to the fourth order in $x=\left(u_{i}-u_{i+1}\right) / \sqrt{m}$, where $u_{i}$ is mass-renormalized displacement for the $i$ th particle. It is a general FPU model with both cubic and quartic interactions. The leads do not have nonlinear interactions. We use the mass of carbon, $m=12 \mathrm{u}$, in the calculation. Thus we can consider the results correspond to that of a carbon chain.

In Fig. 7 we present the mean-field results of conductance $\bar{\kappa}$ as a function of temperature for different lengths of the chain. As we can see, below about $300 \mathrm{~K}$, the conductance is independent of chain lengths; this is the ballistic region of heat conduction. At high tem-

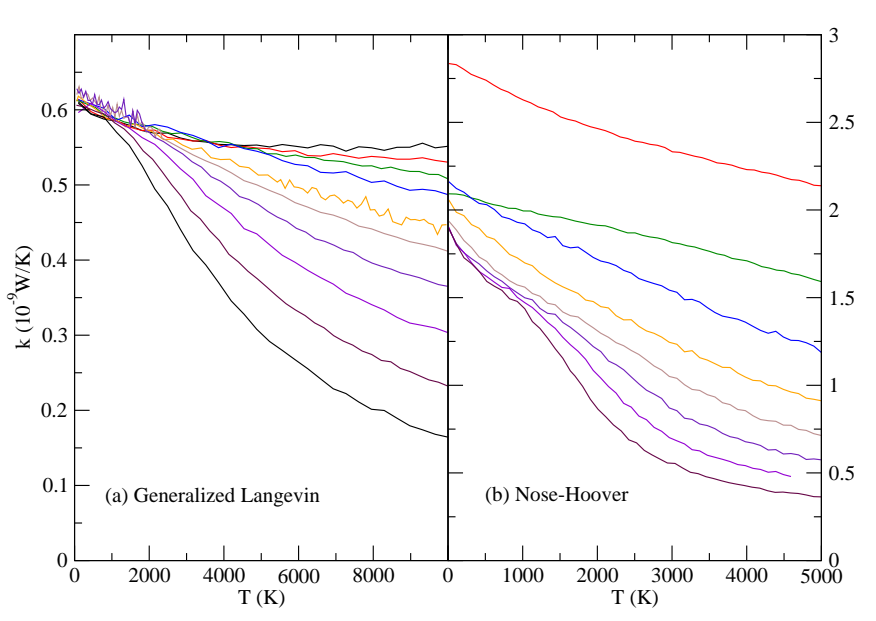

FIG. 8: Molecular dynamics results of the thermal conductance for the model as in Fig. 7 (a) "Generalized Langevin" thermal heat baths from sizes $4,8,16, \ldots, 2048$, from top to bottom. (b) Nosé-Hoover thermostat with size $8,16,32, \ldots$, 1024 .

peratures, the conductance decreases with the system size. For a completely diffusive heat transport, we should have $\bar{\kappa} \propto 1 / L$. We are far from this. Since in FPU model real diffusive behavior is impossible, the reduction in conductance in the classical regime should be $L^{\alpha-1} \approx L^{-0.6}$. The observed exponent for sizes up to 256 is much smaller. In fact, this result is expected because the mean free path in a typical carbon systems (such as carbon nanotubes [40] or diamond) is of order $\mu \mathrm{m}$. Since the conductivity decreases with temperature typically as $1 / T$, even at few thousand kelvin, the mean free path is expected to be 100 to 1000 lattice spacings. Thus we see only tendency towards diffusive behavior. It is also clear that ballistic transport and diffusive transport are at quite different time and length scales. The present calculation is already very large on an IBM 500processor parallel computer. It is out of the possibility to see diffusive behavior for large sizes within the present approach. But this example does give us an indication that the mean-field theory could give diffusive behavior for appropriate systems.

It is instructive to compare the mean-field results with molecular dynamics results. In Fig. 8, we present the MD results with the generalized Langevin-like heat-bath and the Nosé-Hoover heat bath. The colored noise is implemented using a standard spectrum method with fast Fourier transform [41]. It turns out that the results for the thermal conductance is very sensitive to the bath used and detail couplings between the bath and system. It is clear that quantum nature shows up already as high as $1000 \mathrm{~K}$ for this model chain. This can be understood by a very high Debye temperature $T_{D}=\hbar \omega_{\max } / k_{B} \approx 2000 \mathrm{~K}$, where $\omega_{\max }=\sqrt{4 K_{c}}$. The Landauer formula, Eq. (56), provides a rigorous upper bound for the thermal conductance (when $\tilde{T}[\omega]=1$, $T \rightarrow \infty), \bar{\kappa}_{\max }=\frac{1}{2 \pi} k_{B} \omega_{\max } \approx 0.65 \mathrm{nW} / \mathrm{K}$. Interestingly, 


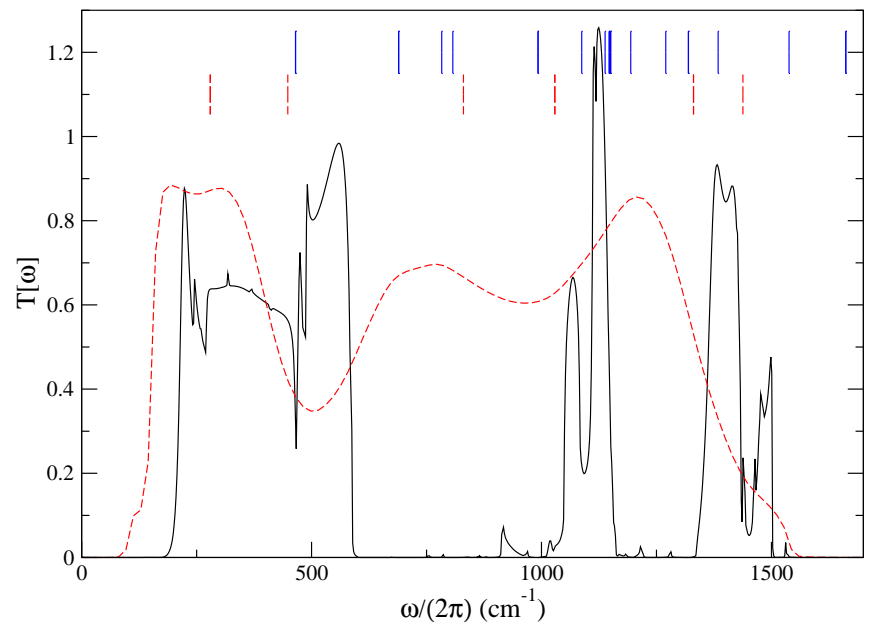

FIG. 9: Effective transmission of the benzene ring junction at a temperature of $300 \mathrm{~K}$. The solid line is for a full model with force constants calculated from Gaussian, while the dotted line is from a simplified 2D model. The vertical bars indicate the vibrational frequencies of isolated benzene rings of the full model (topmost) and simplified model (dashed), respectively.

this is satisfied at low temperatures by the generalized Langevin data.

To what extent the mean-field theory is "correct"? We can see that it is qualitatively correct for the accessible temperature and size ranges. However, if we trust the Langevin heat-bath result, then, it appears that the mean-field approximation somehow over estimates the effect of nonlinearity.

\section{Benzene ring}

Molecular devices have attracted a lot of attentions in recent years, due to their potential for future generation electronics. Experiments have been conducted on such systems; theoretical analyses of their electronic and thermal transport properties have been performed [5, 42].

In this subsection, we examine the thermal transport properties of a benzene molecule connected to two polyethylene leads. Two opposite sites of a benzene ring, which are normally the hydrogen atoms, are now bound covalently to carbon atoms, forming a 1,4-dibutylbenzene molecule representing the whole system. The system has 14 carbon atoms and 22 hydrogen atoms. The interactions are calculated using Gaussian 03 [43]. We use the Hartree-Fock method with the $6-31 \mathrm{G}$ basis set. We have also used a convenient new feature of Gaussian, which can calculate anharmonic interactions up to the 4th order.

Fig. 9 (solid line) shows the effective transmission function $\tilde{T}[\omega]$ of the benzene junction model at a temperature of $300 \mathrm{~K}$ calculated using the mean-field theory. The peak features are complicated, resulting from the vibrational modes of the molecule, the interaction with the leads, and the vibrational spectra of the leads, as well as the temperature effect. To understand the transmission function better, we correlate the peak features with the vibrational spectrum of an isolated benzene molecule calculated from Gaussian, shown in Fig. 9 as solid bars. The transmission peaks are roughly located at where the vibrational frequencies appear, but somewhat shifted to low frequencies. It is difficult to identify the peaks with eigenmodes unambiguously.

We notice that the hydrogen atoms do not play much role in the thermal transport. In particular, there are frequencies around $3000 \mathrm{~cm}^{-1}$ associated with the vibrations of $\mathrm{H}$ atoms, but there is no transmission in these high frequencies. Vibrations of $\mathrm{H}$ atoms have only local effects. We confirm that the $\mathrm{H}$ atoms are less important by a much simplified model retaining only the degrees of freedom of the carbon atoms in a $2 \mathrm{D}$ model. The leads are pseudo polyethylene chains; we use an united atom approximation, so the $\mathrm{CH}_{2}$ group is treated as a single atom. The force constant between each atom is obtained from the DREIDING model [44]. There are only bond interactions in the leads, and we have used the Morse function to calculate the bond potential, which has the form $D\left(e^{-\alpha\left(R-R_{0}\right)}-1\right)^{2}$, where $D=70(\mathrm{kcal} / \mathrm{mol})$, $\alpha=\sqrt{5} \AA^{-1}$, and $R-R_{0}$ is the displacement (with the equilibrium bond length $R_{0}=1.4 \AA$ ). For the benzene ring, we also include an angular potential, which has the form $\frac{4}{3}\left(\cos \left(\theta_{i j k}\right)+\frac{1}{2}\right) E$, where $E=100(\mathrm{kcal} / \mathrm{mol})$. We make a Taylor expansion of the potential and calculate the force constants up to the 4 th order.

The transmission for the simplified model (dotted line in Fig. 9) is smoother due to much reduced degrees of freedom. It is qualitatively in agreement with the full model. In this case, the peaks appear to be in good agreement with the vibrational modes of a hexagonal ring.

\section{Carbon nanotube junction}

Thermal transport of carbon nanotubes has been studied by molecular dynamics [45, 46, 47, 48], Landauer formula ballistic transport [13], and a phenomenological theory taking into account the nonlinear effect [49]. There are also experimental works on carbon nanotubes 50]. We have also studied the heat transport properties of the $(5,0)$ carbon nanotube using a tight-binding (TB) method [51], of which the TB parameters for the carbon atom have been checked recently [52]. The left lead, the central junction, and the right lead consist of forty, twenty, and forty atoms, respectively. A unit cell of the $(5,0)$ nanotube has twenty atoms. The tube's axial dimension is long enough (about $20 \AA$ ) to allow a single $\Gamma$ point to be used for the $k$-point sampling. We have written a force subroutine in order to perform atomic relaxation of the nanotube as well as to calculate force constants by numerical finite differences. The spring constants $K^{\alpha}$, the nonlinear force constants $T_{i j k}$ and $T_{i j k l}$ are systematically obtained by displacing one, 


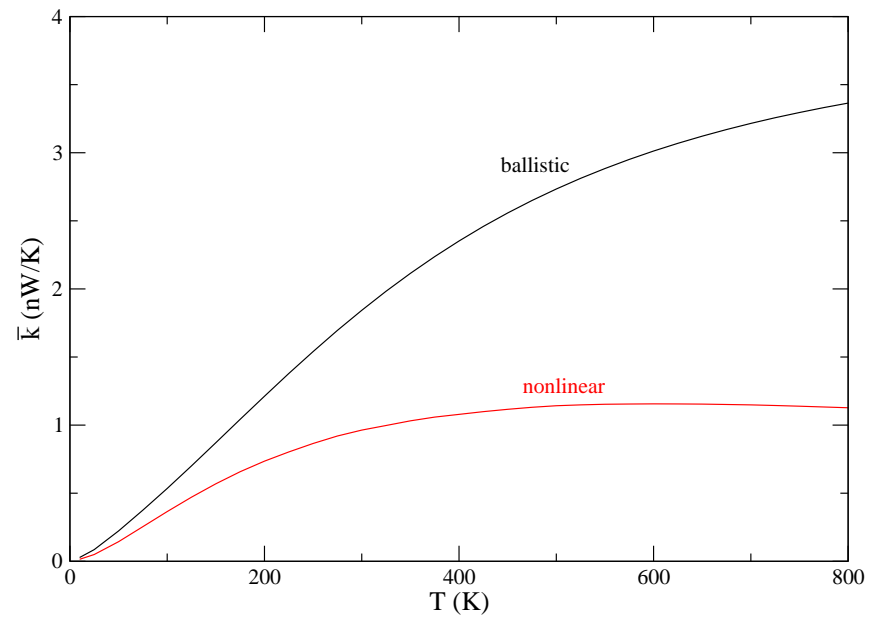

FIG. 10: Thermal conductance $\bar{\kappa}$ as a function of temperature $T$ for a twenty-atom $(5,0)$ carbon nanotube junction.

two, and three atoms, respectively. For each displacement, the forces acting on the atoms are used in the central-difference scheme to calculate the force constants. Since the TB method we have adopted is free from numerical noises, we have used a reasonably small maximum displacement of $h_{\max }$ of $3 \times 10^{-4} \AA$ (i.e., the atoms are displaced not more than $h_{\max }$ from their equilibrium positions). We note that this value of $h_{\max }$ is two orders of magnitude smaller than that commonly used in a supercell force-constant method [53, 54].

In Fig. 10 we present the results of thermal conductance calculations. We find that for such a complicated system, the mean-field iterations are hardly converging, thus we only give the ballistic (where the nonlinear interactions are turned off), and a first-order perturbative result using the (single-line) diagrams in Fig. 4. It is clear that the nonlinear interactions have a large effect even at moderately high temperatures. The thermal conductance is slightly peaked around $600 \mathrm{~K}$ for the nonlinear result. We expect that the result should be reliable at least up to room temperatures.

\section{CONCLUSION}

We presented a nonequilibrium Green's function method for thermal transport in nano-junctions. We gave a unique derivation of the equations of motion of Green's functions and rules for generating and computing Feynman diagrams in a contour-ordered Green's function setting. The nonlinear interaction was treated with a systematic perturbative expansion for self-energies. For practical calculations, the mean-field approximation has to be adopted. We are still left with a technical difficulty that the mean-field equations sometimes do not converge. Several versions of computer programs have been written for consistency checks (a Mathematica notebook for an initial fast prototyping, a Fortran program with MPI for very general models, and a specialized program for the $1 \mathrm{D}$ cubic onsite model). We have applied the programs to a number of model systems. Several sophisticated 'first-principles' methods were used in the force-constant calculations for realistic models. For the FPU model, we can accurately predict the ballistic thermal transport and crossover to diffusive behavior at sufficiently high temperatures. We proposed to use generalized Langevin heat baths, which are more appropriate for nanojunctions. For the benzene ring model, we observed interesting behaviors in the transmission function related to the atomic vibrational modes of the molecule. For carbon nanotubes, it is clear that nonlinear effect is quite strong even at low temperatures.

The Green's function discussed here is more fundamental than the phonon distribution function in the Boltzmann equation. The connection of the present formulation with the quantum Boltzmann equation or the phonon Boltzmann equation is something worth pursuing. Formally, they are related by the Wigner transformation. If we can reduce the matrix of the Green's functions to vector of distribution functions (in momentum space), potentially much larger systems can be treated if we are willing to forsake some atomic details. Clearly, the nonequilibrium Green's function method is at its best for small nanoscale systems. A combination of the present method with electronic transport and electron-phonon interactions is worthy of further explorations.

\section{Acknowledgements}

We thank Lin Yi for introducing us the nonequilibrium Green's function method. We also thank Hong Guo, Qinfeng Sun, Sai Kong Chin, and Jingtao Lü for discussions. C. K. G. thanks D. A. Papaconstantopoulos, L. Shi, and M. J. Mehl for a copy of the tight-binding program static and various initial help with the use of the program. This work was supported in part by a National University of Singapore Academic Research Fund. The computations were carried out on the Institute of High Performance Computing IBM clusters and on the Singapore-MIT Alliance Opteron cluster.
[1] R. E. Peierls, Quantum Theory of Solids (Oxford University Press, 1955), Chap. 2.

[2] G. P. Srivastava, The Physics of Phonons (Adam Hilger,
Bristol, 1990).

[3] P. Carruthers, Rev. Mod. Phys. 33, 92 (1961).

[4] D. G. Cahill, W. K. Ford, K. E. Goodson, G. D. Mahan, 
A. Majumdar, H. J. Maris, R. Merlin, and S. R. Phillpot, J. Appl. Phys. 93, 793 (2003).

[5] M. Galperin, M. A. Ratner, and A. Nitzan, cond-mat/0612085

[6] L. G. C. Rego and G. Kirczenow, Phys. Rev. Lett. 81, 232 (1998); M. P. Blencowe, Phys. Rev. B 59, 4992 (1999).

[7] M. Michel, G. Mahler, and J. Gemmer, Phys. Rev. Lett. 95, 180602 (2005); M. Michel, J. Gemmer, and G. Mahler, Int. J. Mod. Phys. B 20, 4855 (2006).

[8] D. Segal, A. Nitzan, and P. Hänggi, J. Chem. Phys. 119, 6840 (2003); D. Segal and A. Nitzan, Phys. Rev. Lett. 94, 034301 (2005).

[9] G. Chen, Phys. Rev. Lett. 86, 2297 (2001).

[10] C. V. D. R. Anderson and K. K. Tamma, Phys. Rev. Lett. 96, 184301 (2006).

[11] A. Ozpineci and S. Ciraci, Phys. Rev. B 63, 125415 (2001).

[12] N. Mingo and L. Yang, Phys. Rev. B 68, 245406 (2003).

[13] T. Yamamoto and K. Watanabe, Phys. Rev. Lett, 96 255503 (2006).

[14] A. Dhar and D. Sen, Phys. Rev. B 73, 085119 (2006).

[15] J.-S. Wang, J. Wang, and N. Zeng, Phys. Rev. B 74, 033408 (2006).

[16] N. Mingo, Phys. Rev. B 74, 125402 (2006).

[17] Y. Meir and N. S. Wingreen, Phys. Rev. Lett. 68, 2512 (1992); A.-P. Jauho, N. S. Wingreen, and Y. Meir, Phys. Rev. B 50, 5528 (1994).

[18] H. Haug and A.-P. Jauho, Quantum Kinetics in Transport and Optics of Semiconductors (Springer, Berlin, 1996).

[19] L. Kadanoff and G. Baymn, Quantum Statistical Mechanics (W. A. Benjamin, New York, 1962).

[20] S. Doniach and E. H. Sondheimer, Green's Functions for Solid State Physicists (W. A. Benjamin, Reading, 1974), Chap. 1.

[21] G. D. Mahan, Many Particle Physics, 3rd Ed. (Springer, New York, 2000).

[22] C. Niu, D. L. Lin, and T.-H. Lin, J. Phys.: Condens. Matter, 11, 1511 (1999).

[23] L. V. Keldysh, Sov. Phys. JETP, 20, 1018 (1965).

[24] A. L. Fetter and J. D. Walecka, Quantum Theory of Many-Particle Systems (McGraw-Hill, New York, 1971).

[25] R. G. D. Valle and P. Procacci, Phys. Rev. B 46, 6141 (1992).

[26] D. C. Langreth, in Linear and Nonlinear Electron Transport in Solids, edited by J. T. Devreese and E. van Doren (Plenum, New York, 1976), pp.3-32.

[27] R. Landauer, IBM J. Res. Dev. 1, 223 (1957); Phil. Mag. 21, 863 (1970).

[28] C. Caroli, R. Combescot, P. Nozieres, and D. SaintJames, J. Phys. C 4, 916 (1971).

[29] A. Dhar and D. Roy, J. Stat. Phys. 125, 801 (2006); see also A. Dhar and K. Wagh, cond-mat/0604170

[30] K. Lindenberg and B. J. West, The Nonequilibrium Sta- tistical Mechanics of Open and Closed Systems (VCH Publishers, New York, 1990), p.194.

[31] M. P. López Sancho, J. M. López Sancho, and J. Rubio, J. Phys. F: Met. Phys. 15, 851 (1985).

[32] H. Spohn, math-ph/0505025 K. Aoki, J. Lukkarinen, H. Spohn, J. Stat. Phys. 124, 1105 (2006).

[33] Focus issue: the "Fermi-Pasta-Ulam" problem - the first 50 years. Chaos, 15, (2005).

[34] S. Lepri, R. Livi, and A. Politi, Phys. Rep. 377, 1 (2003).

[35] B. Li, J. Wang, L. Wang, and G. Zhang, Chaos, 15, 015121 (2005).

[36] S. Lepri, R. Livi, and A. Politi, Europhys. Lett. 43, 271 (1998).

[37] S. Lepri, Phys. Rev. E 58, 7165 (1998).

[38] O. Narayan and S. Ramaswamy, Phys. Rev. Lett. 89, 200601 (2002); T. Mai, A. Dhar, O. Narayan, cond-mat/0608034

[39] L. Delfini, S. Lepri, R. Livi, and A. Politi, Phys. Rev. E 73, 060201(R) (2006); cond-mat/0611278

[40] Y. Xiao, X. H. Yan, J. X. Cao, and J. W. Ding, J. Phys: Condens. Matter, 15, L341 (2003).

[41] J. García-Ojalvo and J. M. Sancho, Noise in Spatially Extended Systems (Springer-Verlag, New York, 1999).

[42] A. Buldum, D. M. Leitner, and S. Ciraci, Europhys. Lett. 47, 208 (1999).

[43] M. J. Frisch, et al., Gaussian 03, Revision C.02, (Gaussian Inc., Wallingford, 2004).

[44] S. L. Mayo, B. D. Olafson, and W. A. Goddard III, J. Phys. Chem. 94, 8897 (1990).

[45] S. Berber, Y.-K. Kwon, and D. Tománek, Phys. Rev. Lett. 84, 4613 (2000).

[46] S. Maruyama, Physica B 323, 193 (2002).

[47] Z. Yao, J.-S. Wang, B. Li, and G.-R. Liu, Phys. Rev. B 71, 085417 (2005).

[48] G. Zheng and B. Li, J. Chem. Phys. 123, 114714 (2005).

[49] J. Wang and J.-S. Wang, Appl. Phys. Lett. 88, 111909 (2006).

[50] P. Kim, L. Shi, A. Majumdar, and P. L. McEuen, Phys. Rev. Lett. 87, 215502, (2001); E. Pop, D. Mann, Q. Wang, K. Goodson, and H. Dai, Nano Letters, 6, 96 (2006).

[51] R. E. Cohen, M. J. Mehl, and D. A. Papaconstantopoulos, Phys. Rev. B 50, 14694 (1994); M. J. Mehl and D. A. Papaconstantopoulos, Phys. Rev. B 54, 4519 (1996); D. A. Papaconstantopoulos, M. J. Mehl, S. C. Erwin, and M. R. Pederson, Mater. Res. Soc. Proc. 491, 221 (1998).

[52] M. G. Fyta, I. N. Remediakis, P. C. Kelires, and D. A. Papaconstantopoulos, Phys. Rev. Lett. 96, 185503 (2006).

[53] C. K. Gan, Y. P. Feng, and D. J. Srolovitz, Phys. Rev. B 73, 235214 (2006).

[54] K. Parlinski and Y. Kawazoe, Phys. Rev. B 60, 15511 (1999). 